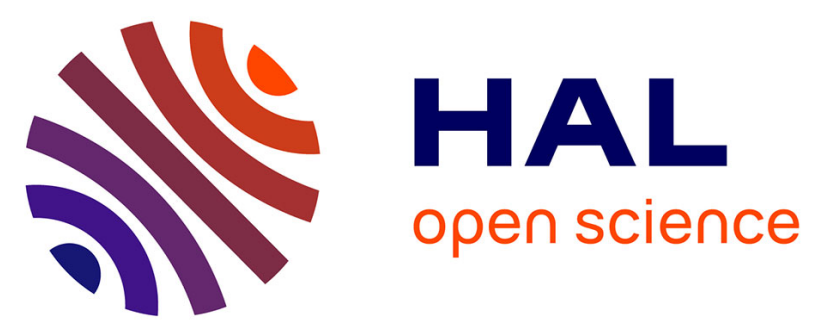

\title{
Repetitive control of an XYZ piezo-stage for faster nano-scanning: Numerical simulations and experiments
}

S. Necipoglu, S. A. Cebeci, Cagatay Basdogan, Y. E. Has, L. Guvenc

\section{To cite this version:}

S. Necipoglu, S. A. Cebeci, Cagatay Basdogan, Y. E. Has, L. Guvenc. Repetitive control of an XYZ piezo-stage for faster nano-scanning: Numerical simulations and experiments. Mechatronics, 2011, 21 (6), pp.1098-1107. 10.1016/j.mechatronics.2011.06.004 . hal-03177415

\section{HAL Id: hal-03177415 https://hal.science/hal-03177415}

Submitted on 23 Mar 2021

HAL is a multi-disciplinary open access archive for the deposit and dissemination of scientific research documents, whether they are published or not. The documents may come from teaching and research institutions in France or abroad, or from public or private research centers.
L'archive ouverte pluridisciplinaire HAL, est destinée au dépôt et à la diffusion de documents scientifiques de niveau recherche, publiés ou non, émanant des établissements d'enseignement et de recherche français ou étrangers, des laboratoires publics ou privés. 


\title{
Repetitive control of an XYZ piezo-stage for faster nano- scanning: numerical simulations and experiments
}

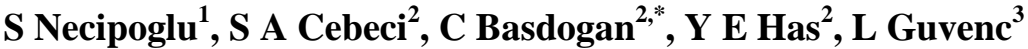 \\ ${ }^{1}$ Department of Mechanical Engineering, Istanbul Technical University, Istanbul, 34437, \\ Turkey. \\ ${ }^{2}$ College of Engineering, Koc University, Istanbul, 34450, Turkey. \\ ${ }^{3}$ Department of Mechanical Engineering, Okan University, Istanbul, Turkey.
}

\begin{abstract}
A Repetitive Controller (RC) is implemented to control the z-axis movements of a piezo-scanner used for AFM scanning and then tested through scan experiments and numerical simulations. The experimental and simulation results show that the RC compensates phase delays better than the standard PI controller at high scan speeds, which leads to less scan error and lower interaction forces between the scanning probe and the surface being scanned. Since the AFM experiments are not perfectly repeatable in the physical world, the optimum phase compensators of the RC resulting this performance are determined through the numerical simulations performed in MATLAB/Simulink. Furthermore, the numerical simulations are also performed to show that the proposed RC is robust and does not require re-tuning of these compensators when the consecutive scan lines are not similar and a change occurs in the probe characteristics.
\end{abstract}

\footnotetext{
* Corresponding Author: College of Engineering, Koc University, Istanbul, 34450, Turkey Tel: $90+2123381721$ Fax: 90+212338 1548 cbasdogan@ku.edu.tr
} 


\section{Introduction}

Increasing the scan speed of an AFM without sacrificing the scan quality is an active area of research. One method of increasing the scan speed of an AFM is by improving the mechanical design of the scanner. In this context, the goal is to increase the resonance frequency of the scanner so that the scanner has a larger bandwidth, allowing higher speeds to be achieved. For this purpose, a two-layered scanner architecture is proposed in [1], which guarantees decoupling of the $x$ and $y$ axes and provides faster z-axis motion. A fast scanner is designed in [2] by pushing the performance of each individual part of electronics to its limit: a preamplifier with a bandwidth of $600 \mathrm{kHz}$, a feedback electronics with a bandwidth of $1 \mathrm{MHz}$, a home-built bus structure for the fast data transfer, fast analog to digital converters, and low-noise drivers. The scanner proposed in [3] can achieve scanning speeds that are more than two orders of magnitude faster than the current commercial AFM systems. The new design is based on piezoelectric stack actuators and a flexure mechanism that enables decoupling of the different axes of motion while keeping the mechanical structure stiff. Moreover, in order to reach high resonance frequencies (and hence high scan speeds) in all positioning directions, the mechanical paths are kept as short as possible. In [4], a high resonance frequency of $540 \mathrm{kHz}$ is achieved for the z-actuator using a support mechanism, what the authors call as "inertia balance". In this method, a cubic piezo actuator enabling the z-motion is supported at the four sides perpendicular to the extension axis to increase its resonance frequency.

The other method for increasing the scan speed of an AFM is to replace the conventional PI controller used in the feedback loop with a more sophisticated one (see the review of the control literature in [5], [6], and [7]). In this study, we focus on the latter method and propose a repetitive controller (RC) for faster scanning of nano-scale surfaces in tapping mode AFM. The existing control studies on AFM can be categorized into two groups: a) those aiming to control the scanning probe and b) those focusing on the control of the scanner (i.e. the piezo actuator holding the sample). The latter group can be further divided into two sub-groups: controllers designed to improve the scan speed by adjusting the periodic lateral movements of the scanner on $\mathrm{X}-\mathrm{Y}$ plane and those designed to maintain 
the image quality at high scan speeds by adjusting the vertical movements of the scanner along the Zaxis. In Figure 1, the movements of the scanner along the $\mathrm{X}, \mathrm{Y}$ and $\mathrm{Z}$ axes during an AFM scan are illustrated. Typically, the scanner is moved back and forth on the X-axis by sending a triangular voltage signal to the $\mathrm{X}$-actuator while it is slowly advanced along the $\mathrm{Y}$ axis by sending a ramp voltage signal to the $\mathrm{Y}$-actuator. The resulting scan motion is a triangular trajectory on the $\mathrm{X}-\mathrm{Y}$ plane. Obviously, tracking a periodic back and forth motion is more challenging than tracking a ramp input. Hence, the research in that area is mostly focused on improving the scan speed along the $\mathrm{X}$-axis. Moreover, the controllers used for the lateral axes ( $\mathrm{X}$ and $\mathrm{Y})$ aim to reduce the image distortions that occur due to the nonlinear behavior of the piezo-scanners such as creep, hysteresis, and thermal effects as well as the coupling between them.

a)

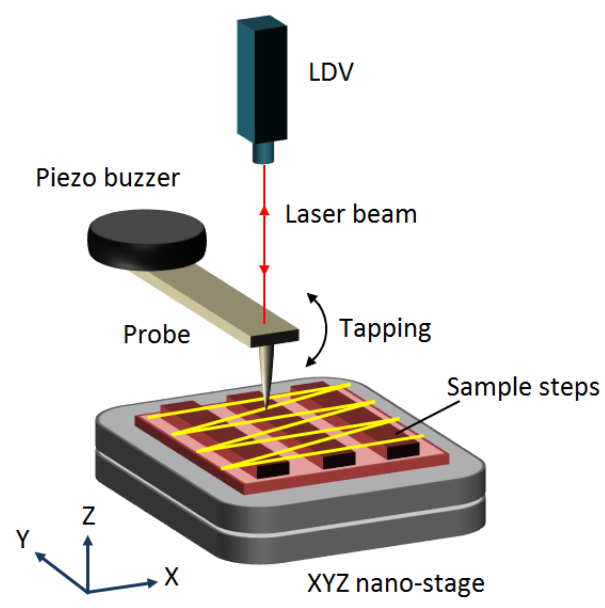

b)
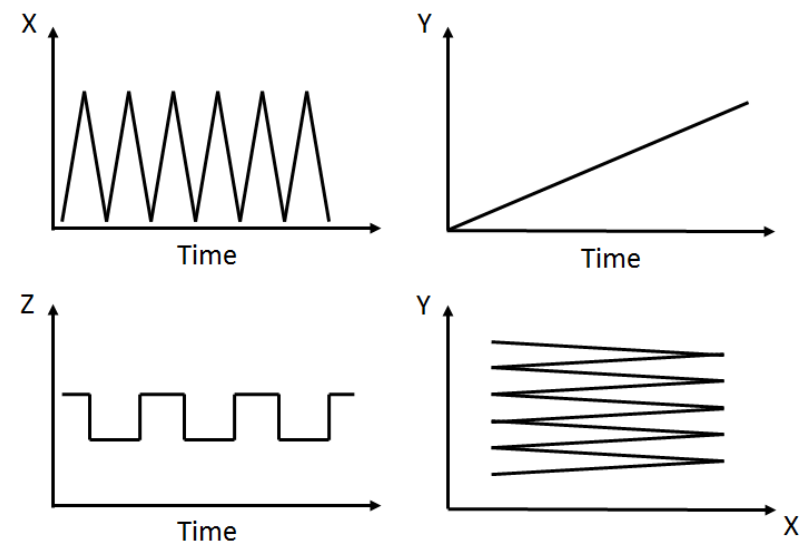

Figure 1. a) Illustration of our AFM setup b) The movements of the scanner during the scanning of nano-scale steps.

Typically, a PI controller is used for controlling the X-axis motion in commercial AFMs. But due to the bandwidth limitation of a standard PI controller, making an improvement in scan speed is limited. For this reason, more sophisticated control techniques have been proposed to achieve higher scan speeds. In [8], inverted models of creep, hysteresis, and induced vibrations are utilized in the controller design to reduce the undesired nonlinear effects. A feedback controller combined with a feedforward controller is proposed in [9] and [10], where the feedback controller is used to 
compensate for the creep and hysteresis while the feedforward controller is used to increase the scan speed. In [11], Hung et al. integrate the feedback and feedforward controllers using a post-fitting control scheme. An inversion based iterative controller is also proposed in [12] to reduce the undesired hysteresis effects and in [13] to reduce the output oscillations via pre-filter. This approach is further improved in [14], where a model-free design is suggested for easier implementation of the controller. A new stage design along with the implementation of a feedback type $H_{\infty}$ controller is proposed in [15] for faster scanning. The authors report that the use of $\mathbf{H}_{\infty}$ controller provides substantial improvements in the positioning speed and precision, while eliminating the undesirable nonlinear effects of the actuator. An iterative learning controller (ILC) is used to compensate for hysteresis-caused positioning errors in AFM scanning [16]. In [17], an ILC is combined with a $\mathrm{H}_{\infty}$ feedback controller and a ratio that determines the frequency range in which feedback or feedforward control should be employed is introduced. In [18], Bhikkaji et al. propose an integral resonant controller for suppressing the resonant mode of the piezo actuator to increase the scanning speed. An $\mathrm{RC}$ is proposed in [19] and [20] to increase the lateral scan speed by taking advantage of the repetitive nature of the trajectory followed by the nanopositioner on the X-Y plane. If the sample placed on the nanopositioner is not perfectly aligned with the actuation directions (i.e. if it is slightly rotated with respect to the $z$-axis), the repetitive tracking errors become non-repetitive and the performance of a standard RC may degrade. In [21], a rotation matrix is incorporated into a standard RC to rotate the scan trajectory and make the tracking errors repetitive again.

Developing a controller for the $\mathrm{Z}$-axis is more challenging than developing one for the $\mathrm{X}$ and $\mathrm{Y}$ axes since the trajectory followed on the $\mathrm{X}-\mathrm{Y}$ plane is periodic and known in advance, whereas the sample surface to be scanned is an unknown disturbance affecting the Z-motion of the nanopositioner. Moreover, the interaction forces between the scanning probe and the surface being scanned are nonlinear, which makes it more difficult to adjust the probe position with respect to the sample surface along the Z-axis. Again, a standard PI controller is typically used for the Z-axis control of nanopositioners though more advanced control techniques have been suggested recently to improve the scan performance. In [22], Schitter et al. utilize an $\mathrm{H}_{\infty}$ controller for the $\mathrm{Z}$-axis piezo-actuator to 
keep the interaction forces between the probe and the sample at a constant value, while the movement of the scanner is simultaneously tracked by a model-based feedforward controller to reduce the scan error at high scan speeds. In [23], an adaptive PID controller is implemented for the Z-axis piezoactuator to modify the PID gains on the fly during the scan process based on the control error. In [24], Fujimoto and Oshima propose a Surface Topography Learning Observer (STLO) to estimate the profile of a scan line in the forward pass and then use it during the backward pass of the same line to reduce the scan error. An ILC working in parallel with an $\mathrm{H}_{\infty}$ feedback controller is developed in [25] to improve the dynamic response of the Z-axis piezo and provide robustness. In their implementation, the control input for tracking the current scan line is generated based on the tracking results of the previous scan line. The control theory behind an ILC is similar to that of an RC, but an ILC requires resetting of the initial conditions at the start of each iteration step for the implementation. Therefore, the implementation of an RC is easier than that of an ILC.

In this paper, we propose an $\mathrm{RC}$ for controlling the $\mathrm{Z}$-axis movements of a piezo-stage. The $\mathrm{RC}$ proposed in $[5,19,20,21]$ is similar to ours, but aims to control the lateral axes of a nanopositioner rather than its Z-axis. Controlling the Z-axis using an $\mathrm{RC}$ is more challenging than that of the lateral axes since the surface topography is constructed based on the vertical movements, which is an unknown exogenous disturbance for the system. An RC is known to work well for systems tracking repetitive inputs or rejecting repetitive disturbances. In AFM scanning, the profiles of the successive scan lines are repetitive since the advancement along the Y-axis is small during the raster scanning. The proposed RC takes advantage of the knowledge of the previous scan line while scanning the current one to achieve a better scan performance than that of a standard PI controller. In comparison to our earlier work in the same area [26], we further investigate the effect of our control design on the scan performance using numerical simulations performed in MATLAB/Simulink for various scan scenarios. Numerical simulations performed in MATLAB/Simulink have been used to investigate the effect of changing Q factor of the probe on the scan performance [27] and imaging parameters on the tip sample forces [28]. Following the design of our RC, we investigate the effect of tunable parameters (i.e. phase compensators) on the scan performance extensively using the numerical simulations. Since 
there are many factors (i. e. temperature, dust, humidity, ground vibration, electrical noise, etc) that affect the AFM scan in the real world settings, the scan results are not perfectly repeatable and an extensive analysis based on the scanning experiments is almost impossible. Numerical simulation is an alternative solution to this problem. In our study, the optimum phase compensators (i.e. tunable parameters) of the RC are determined through the numerical simulations performed in MATLAB/Simulink. Furthermore, the numerical simulations are performed to show that the RC does not require re-tuning of these compensators when a change occurs in the probe characteristics. After tuning our RC based on the results of the numerical simulations, we compare its performance to that of the PI controller through the scanning experiments performed in our AFM set-up.

\section{RC Basics}

$\mathrm{RC}$ is a powerful controller for tracking or rejecting repetitive signals. It is based on the internal model principle which states that the steady state error converges to zero if the generator of the applied reference input is placed on the forward path. A good example is an integrator used in front of a plant in a feedback control loop when the input signal is a step function. RC is simply a memory loop which acts as the generator of any repetitive input (Figure 2a). Therefore, it can track or reject any repetitive signal as long as its period, $\tau_{\mathrm{d}}$, is determined correctly. The effect of a memory loop can be better understood if the Bode plot in Figure $2 \mathrm{~b}$ is inspected. As shown in the figure, the gain tends to infinity at the fundamental frequency and at its harmonics. Hence, if the input signal to be tracked or the disturbance signal to be rejected is repetitive, one can introduce high control gains to the system using an $\mathrm{RC}$, thereby reducing steady state error to zero. 
a)
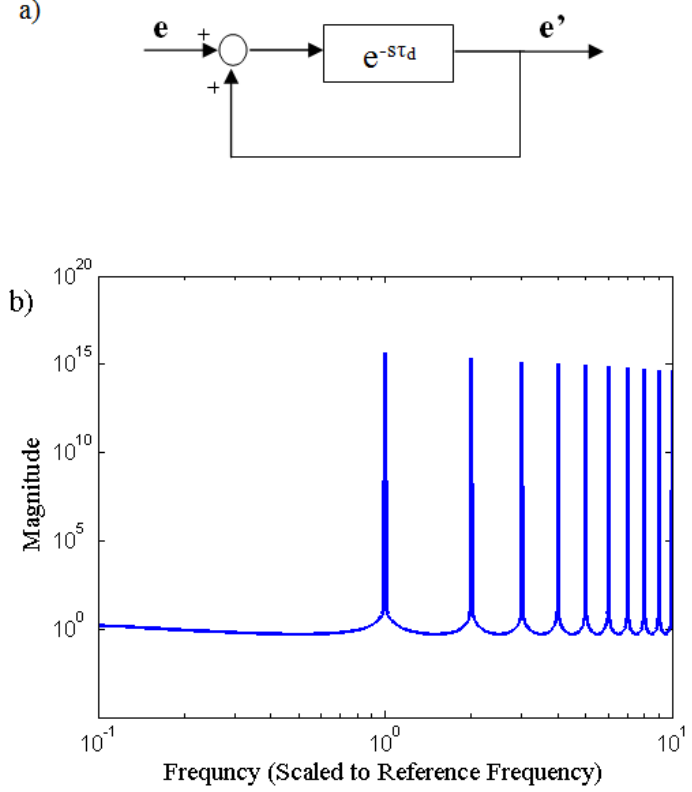

Figure 2. a) The memory loop b) The frequency response of the memory loop.

The block diagram of a typical RC [29] is shown in Figure 3. The design of an RC involves the design of two low-pass filters, $\mathrm{Q}(\mathrm{s})$ and $\mathrm{B}(\mathrm{s})$ along with the selection of two tunable parameters, time advances $\tau_{\mathrm{q}}$ and $\tau_{\mathrm{b}}$. Q(s) is a low-pass filter with a unit d.c. gain, which is utilized to filter out the undesired dynamics at high frequencies and to maintain stability in the presence of uncertainties. Hence, the main purpose of using the Q-filter is to reduce the gain introduced by the RC to the system at the undesired harmonics, which would otherwise lead to instability due to the effects of noise and modeling uncertainties at high frequencies. The design of the Q-filter also affects the operating bandwidth of the RC. The additional phase introduced to the system by the Q-filter can be compensated by a small time advance $\tau_{\mathrm{q}}$ (Figure 3). $\mathrm{B}(\mathrm{s})$ is a low-pass filter used for improving the performance and relative stability of the RC. Consider the regeneration spectrum defined for an RC by Srinivasan and Shaw [30]:

$$
R(\omega)=\left|Q(j \omega)\left[1-B(j \omega) \frac{G(j \omega)}{1+G(j \omega)}\right]\right|
$$


For a stable implementation, the regeneration spectrum, $R(\omega)$, should be less than 1 for all frequencies of $\omega$. Therefore, the B-filter is simply designed to invert the transfer function $G(j \omega) /[1+G(j \omega)]$ at the operating frequencies. The additional phase introduced by the B-filter and the plant can be also compensated by a small time advance, $\tau_{\mathrm{b}}$ (Figure 3). In our implementation, the time advances, $\tau_{\mathrm{q}}$ and $\tau_{\mathrm{b}}$, are tuned for the best performance using the numerical simulations. The effect of these parameters on the system performance is further discussed in Section 4.

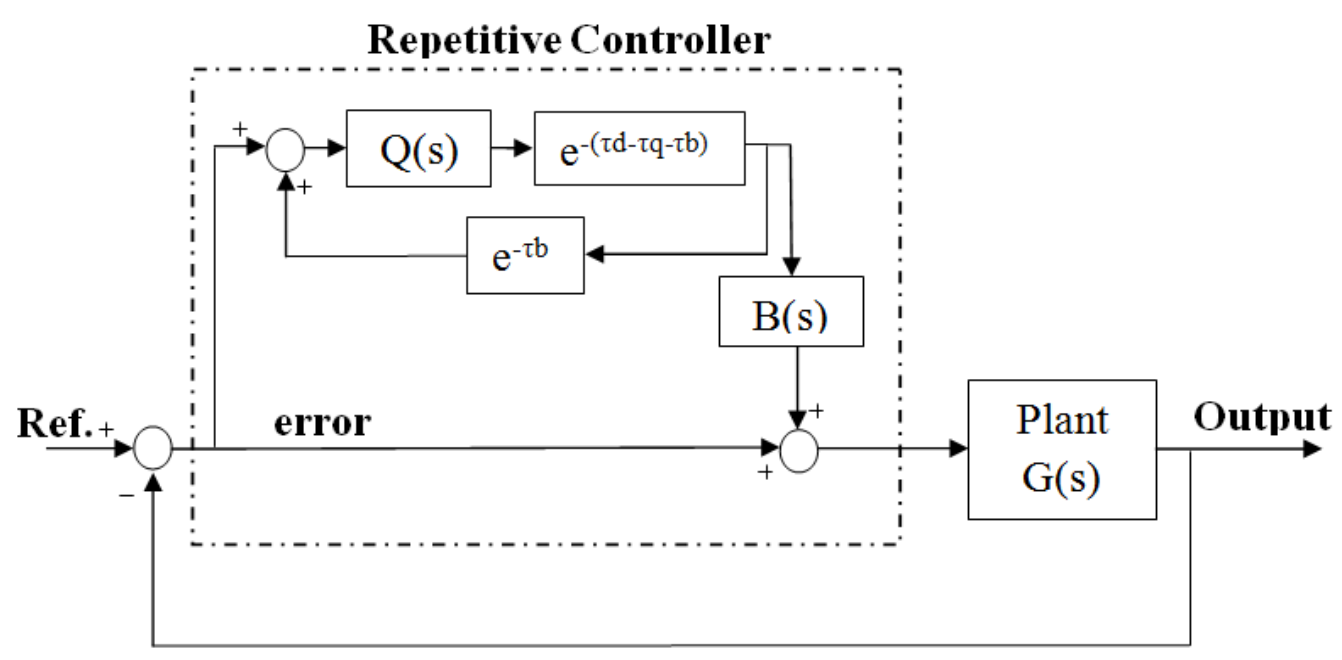

Figure 3. A typical RC scheme.

\section{RC for AFM}

\subsection{Setup}

We have developed a set-up to perform scanning experiments in tapping mode AFM [31] as shown in Figure 4. We have made some modifications on this set-up to implement an RC in series with a standard PI controller as shown in Figure 5. As a result, the tracking errors are corrected by the $\mathrm{RC}$ while the PI controller handles the errors caused by hysteresis, creep, and thermal drift occurring at low-frequencies. The software for the implementation of the controller is developed in LabVIEW. In our set-up (Figure 4), an AFM probe mounted on a piezo-buzzer is brought close to the sample surface via a manual stage for coarse adjustment. The vibrational velocity of the probe tip actuated by the buzzer is measured through a laser doppler vibrometer (LDV). The RMS value of the velocity 
signal is calculated by an analog electronic circuit first, and then transferred to a computer via a data acquisition card.

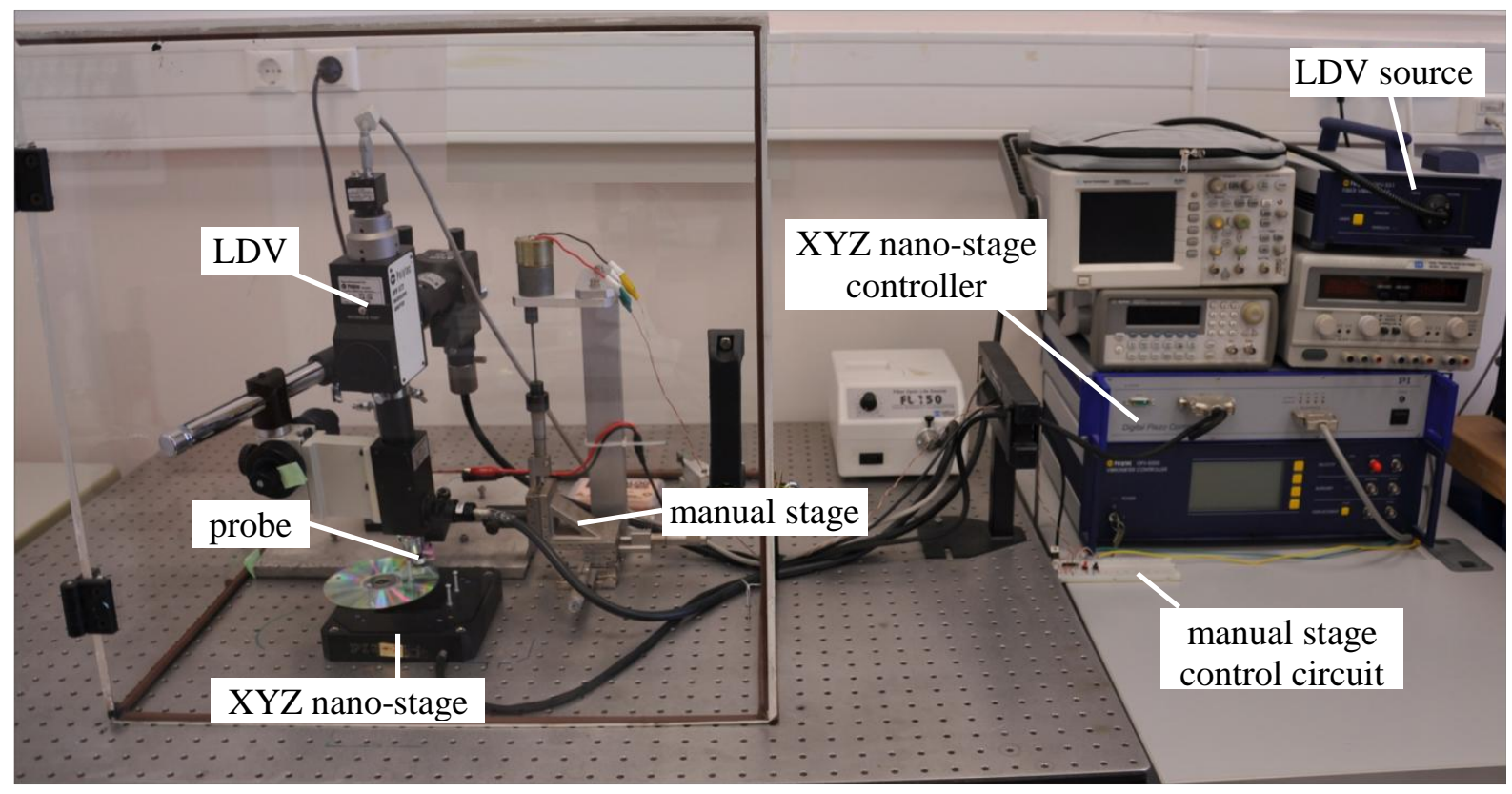

Figure 4. Our AFM set-up.

The vibration amplitude of the probe $\left(\mathrm{A}_{\text {act }}\right)$ is calculated by integrating the RMS velocity, which is then subtracted from the set amplitude $\left(\mathrm{A}_{\text {set }}\right)$ to calculate the error (Figure 5). The error signal is then fed into the controller to generate the control signal, which is sent to the piezo-actuated XYZ nanostage to adjust the tip-sample separation along the Z-axis. This loop runs at $500 \mathrm{~Hz}$. The movements of the nano-stage along the Z-axis are recorded at each grid point on the X-Y plane to obtain the topographical image of the sample surface. 


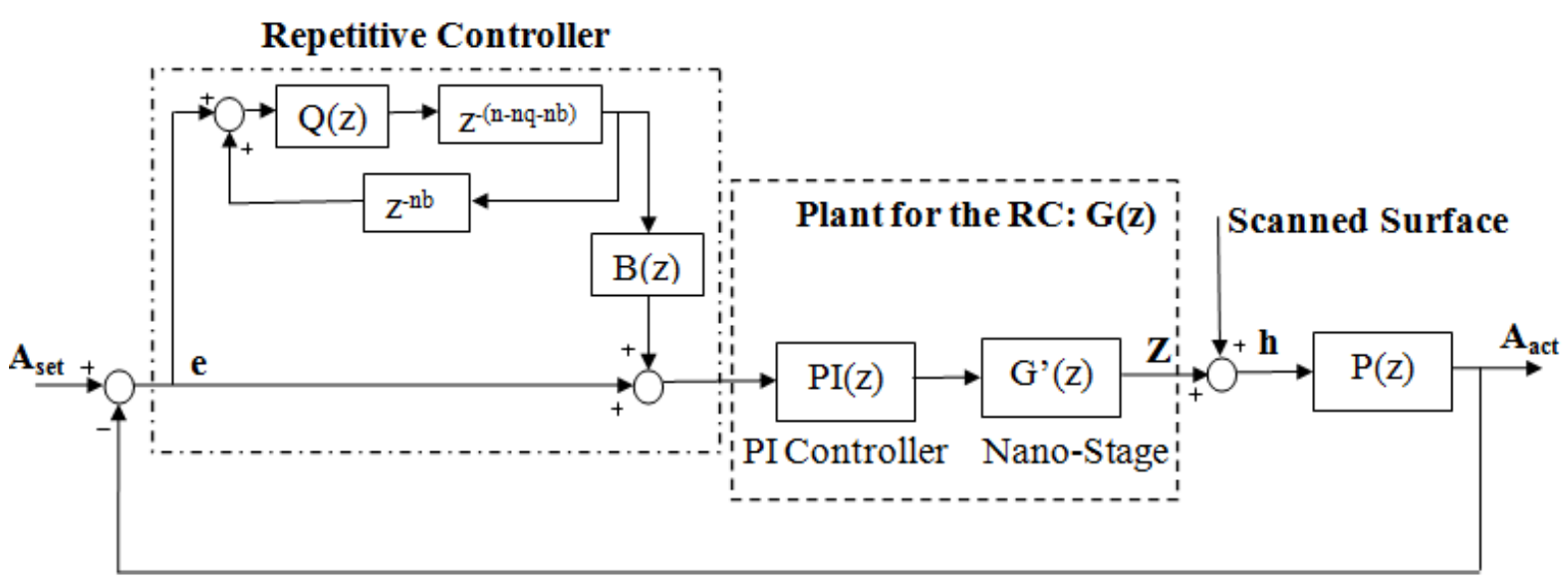

Figure 5. Our implementation of the RC for AFM scanning.

\subsection{System Identification}

An accurate model of the nano-stage is necessary for the proper implementation of the proposed RC. For this purpose, the displacement response of the nano-stage along the Z-axis to an impulse input voltage is recorded and a transfer function model, $G^{\prime}(z)$, is fit to the experimental data using the least squares method. The order of the polynomials in the numerator and the denominator are chosen to be 3 and 7 respectively (see Eq.2). This choice is made by trial and error placing emphasis on the best fit with the minimum degree. Moreover, since the transfer function in Eq. 2 is to be inverted for the implementation of the $\mathbf{R C}$, it has to be minimum phase. The identified transfer function for the stage is

$$
\mathrm{G}^{\prime}(\mathrm{z})=\frac{0.04 \mathrm{z}^{3}+0.07 \mathrm{z}^{2}+0.05 \mathrm{z}+0.01}{\mathrm{z}^{7}-1.34 \mathrm{z}^{6}+1.14 \mathrm{z}^{5}-0.47 \mathrm{z}^{4}+0.04 \mathrm{z}^{3}-0.19 \mathrm{z}^{2}+0.11 \mathrm{z}-0.11}
$$

The frequency responses of the nano-stage and the model are compared in Figure 6 . The frequency response of the nano-stage is obtained by taking the FFT of the experimental impulse response. 


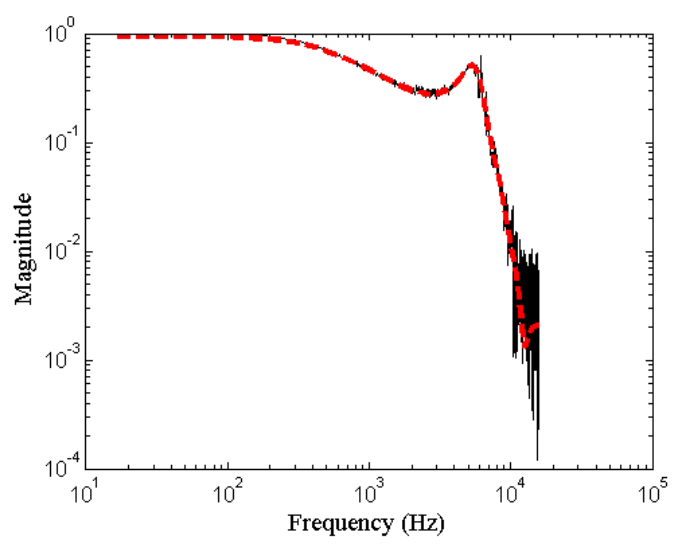

Figure 6. The frequency response of the nano-stage (solid black) and its transfer function model G'(z) (dashed red).

\subsection{Design of the B-filter}

The B filter is designed to be the inverse of the closed loop plant transfer function (see Figure 5), $G(z) /(1+G(z))$, based on the criteria, $R(\omega)<1$, defined in Section 2 . Instead of designing a lowpass filter, a simple static gain is used in [19]. The unmodeled dynamics enforce the authors of [19] to make a conservative selection for the gain value in order to maintain the stability of the system. However, a low-pass filter ensures the stability for a wide range of frequencies. On the other hand, the inverse of $\mathrm{G}(\mathrm{z}) /(1+\mathrm{G}(\mathrm{z}))$ is non-causal since its numerator has a lower degree than its denominator. To eliminate this problem, the inverse of $\mathrm{G}(\mathrm{z}) /(1+\mathrm{G}(\mathrm{z}))$ is multiplied by another $4^{\text {th }}$ order low pass filter having a large bandwidth. The resulting B-filter is expressed in Eq. 3

$$
\mathrm{B}(\mathrm{z})=\frac{\mathrm{z}^{8}-2.34 \mathrm{z}^{7}+2.484 \mathrm{z}^{6}-1.615 \mathrm{z}^{5}+0.554 \mathrm{z}^{4}-0.18 \mathrm{z}^{3}+0.294 \mathrm{z}^{2}-0.261 \mathrm{z}+0.098}{0.047 \mathrm{z}^{8}+0.045 \mathrm{z}^{7}-0.0077 \mathrm{z}^{6}-0.038 \mathrm{z}^{5}-0.012 \mathrm{z}^{4}}
$$

\subsection{Design of the Q-filter}

In order to suppress the undesired harmonics at high frequencies, the bandwidth of the Q-filter must be large. As the bandwidth of the Q-filter increases, the $\mathrm{RC}$ is able to respond faster, but with the cost of additional noise in the control signal. For this reason, we have selected a $4^{\text {th }}$ order model for the Q filter (our numerical simulations show that no significant improvement is obtained when a higher 
order model is used) and experimented with two different bandwidths: one close to the bandwidth of the stage $\left(\omega_{\mathrm{q}}=80 \mathrm{~Hz}, \mathrm{Eq} .4 \mathrm{a}\right)$ and the other is twice the bandwidth of the stage $\left(\omega_{\mathrm{q}}=160 \mathrm{~Hz}, \mathrm{Eq} .4 \mathrm{~b}\right)$.

$$
\begin{aligned}
& \mathrm{Q}_{1}(\mathrm{z})=\frac{8.853 \times 10^{-5}}{\mathrm{z}^{4}-3.612 \mathrm{z}^{3}+4.892 \mathrm{z}^{2}-2.945 \mathrm{z}+0.6649},\left(\text { for } \omega_{\mathrm{q}}=80 \mathrm{~Hz}\right) \\
& \mathrm{Q}_{2}(\mathrm{z})=\frac{0.0012}{\mathrm{z}^{4}-3.262 \mathrm{z}^{3}+3.989 \mathrm{z}^{2}-2.169 \mathrm{z}+0.4421},\left(\text { for } \omega_{\mathrm{q}}=160 \mathrm{~Hz}\right)
\end{aligned}
$$

\subsection{Time advances (phase compensators)}

The time advances $\tau_{\mathrm{q}}$ and $\tau_{\mathrm{b}}$ shown in Figure 3 are used to compensate for the phase delays caused by the filters $\mathrm{Q}$ and $\mathrm{B}$ as well as the other delays caused by the sampling rate and the measurements. Since the transfer functions of the Q and B filters are known in advance, the initial values for these phase compensators can be estimated in advance from the phase diagrams of the filters first and then fine-tuned based on the scan performance. Since our implementation is in discrete domain, both time advances are converted to sample advances by $n=\tau / T_{S}$ where $\mathrm{T}_{\mathrm{S}}$ is the sampling time of the system (Figure 5). Numerical simulations are performed in order to understand the effect of the sample advances, nq and nb, on the performance of our RC.

\section{Simulation Model}

A comprehensive MATLAB/Simulink model (Figure 7) is developed to simulate the whole scan process and test the performance of the RC under different settings of the sample advances nq and nb. For this purpose, our original MATLAB/Simulink model [27] is further improved and modified to implement the proposed RC. 


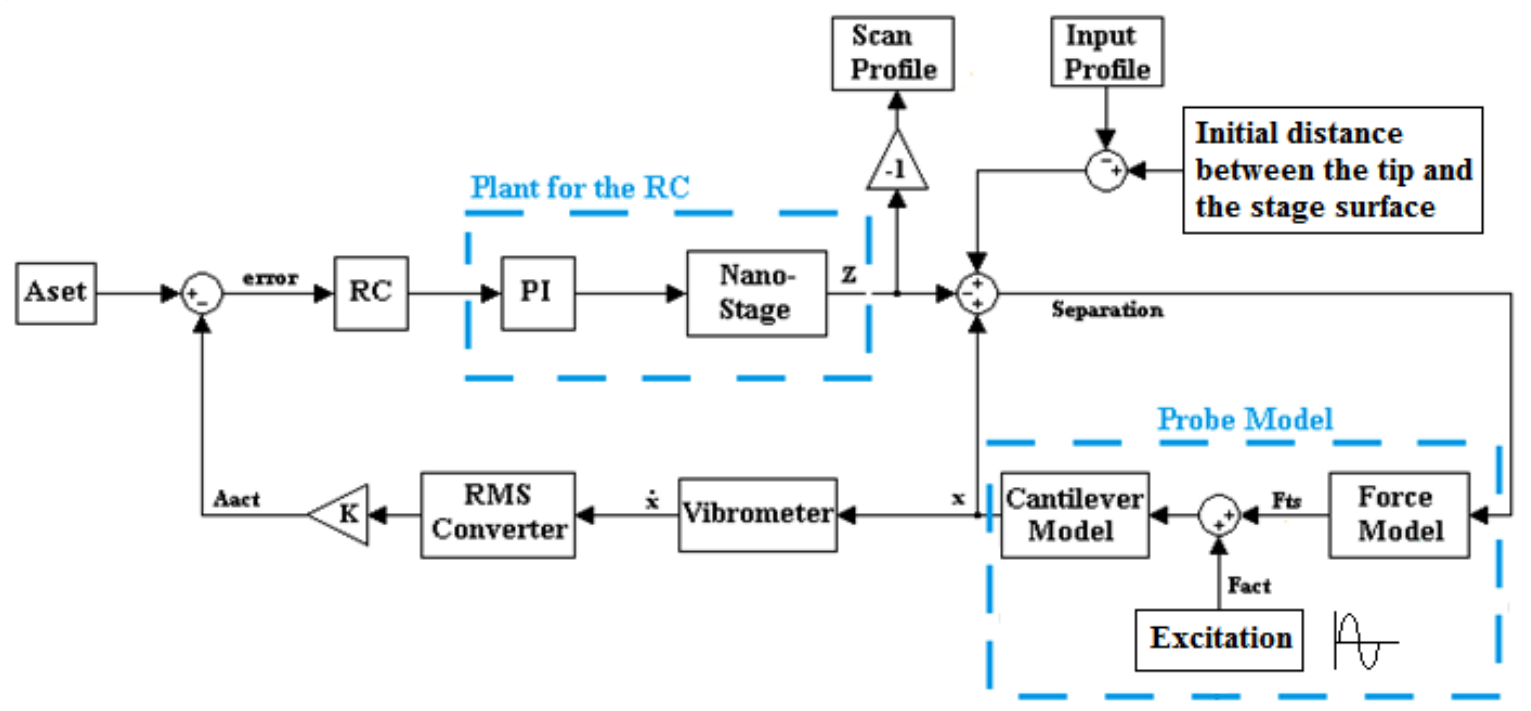

Figure 7. Our MATLAB/Simulink model.

The improvements include the addition of more realistic models for the nano-stage and the interactions between the scanning probe and the sample surface. In our original Simulink model [27], a simple first order model is used for the nano-stage, which is replaced by $G^{\prime}(z)$ in the current study. Moreover, a standard DMT force model was utilized to estimate the nonlinear interaction forces between the AFM probe and the surface being scanned. However, in a standard DMT model, the sticking behavior of the scanning probe to the sample surface is not taken into consideration even though it is frequently observed in AFM scanning. We have improved the DMT model to simulate the sticking effects. For this purpose, we have employed a switch, which decides if the probe sticks to the surface based on the distance between the probe's equilibrium position and the sample surface. When the probe sticks to the surface, the oscillation amplitude of the probe is reduced to zero. Then, in order to separate the probe from the sample surface and hence to bring its vibration amplitude to the desired value, $A_{\text {set }}$, the nano-stage carrying the sample is commanded to move down along the $\mathrm{Z}$-axis by the controller. As a result, the distance between the probe's equilibrium position and the sample surface increases until a threshold distance is reached, and then the probe is assumed to detach from the surface and vibrate in free air again. Here, we assume that the behavior of the probe must be almost linear for our sticking model to work well, which is valid for the stiff probe $(k=42 \mathrm{~N} / \mathrm{m})$ used in our experiments. To test our assumption, a simple experiment with two different probes having nominal 
spring constants of $42 \mathrm{~N} / \mathrm{m}$ (stiff probe) and $3 \mathrm{~N} / \mathrm{m}$ (soft probe) are performed in our AFM setup. During the experiments, the probes are actuated at their resonance frequency and the distance between the probe tip and the sample surface is slowly decreased. The results of the experiments are shown in Figure 8 . The results validate our assumption that the probe with the lower spring constant undergoes a rapid decrease in amplitude while approaching the sample surface and a rapid increase in amplitude while retracting from the same surface. On the other hand, the stiffer probe does not exhibit such behavior. Moreover, the softer probe shows more hysteresis. Therefore, it is reasonable to conclude that the stiff probe shows a more linear behavior than a soft one and the proposed model for sticking effects can be utilized safely in the numerical simulations.
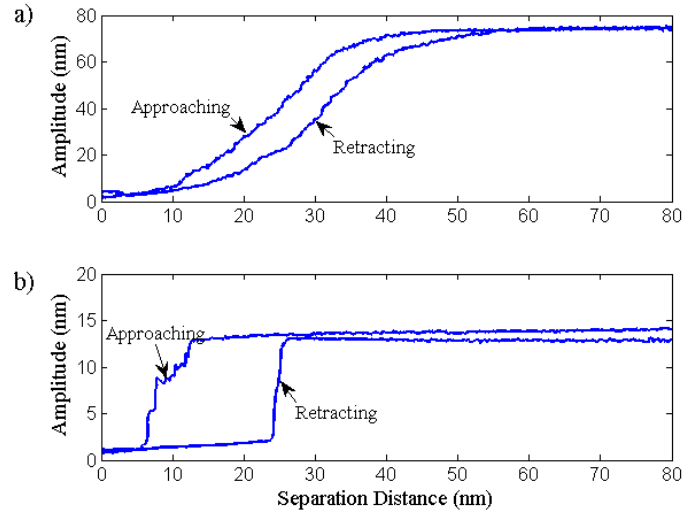

Figure 8. The sticking behavior of a) stiff and b) soft probes during the approaching and retracting phases.

\section{Scanning Simulations}

We investigate the scan performance of our RC using numerical simulations. Since the scan performance, in general, depends on many factors (temperature, humidity, ground vibrations, electrical and environmental noise, etc.) which are not under the full control of an experimenter, we believe that the performance of a new controller for an AFM cannot be fully appreciated without performing extensive numerical simulations.

We first investigate the effect of sample advances, nq and nb, on the performance of RC. The sample advances adjust the phase of the control signal in RC. Therefore, proper tuning of these 
parameters is necessary in order to reduce the phase delays that occur during the actual scanning. For this purpose, we perform scanning simulations and investigate the effect of sample advances on the scan performance in the range of 0 to 15 by incrementing their values by one. We search for the optimum values returning the minimum scan error when a step profile having a width of $\omega_{\mathrm{s}}$ and a height of $h_{S}$ is scanned. The scan error is calculated by integrating the positional tracking error, $e_{\mathrm{X}}$ over the step width, and normalized by the step dimensions as

$$
e_{s}=\frac{\int_{0}^{\omega_{s}}\left|e_{x}\right| d x}{\omega_{s} h_{s}}
$$

The iso-error curves obtained at the scan speed of $3 \mu \mathrm{m} / \mathrm{s}$ are presented in Figure 9. One can observe from the figure that the scan error is minimum when $n q=9$ and $n b=6$.

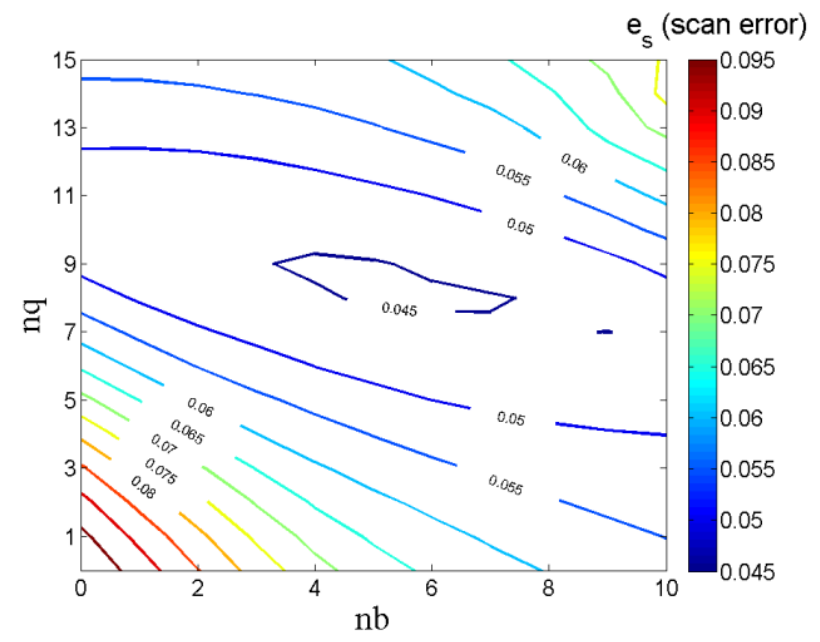

Figure 9. Iso-error curves are generated to investigate the tracking performance of the RC by varying the sample advance parameters.

The scan performance of the $\mathrm{RC}$ for different values of $\mathrm{nq}$ and $\mathrm{nb}$ is shown in Figure 10. As shown in the figure, the sample advance nq influences the upper left and lower right corners whereas the sample advance nb influences the lower left and the upper right corners of the step. 
a)

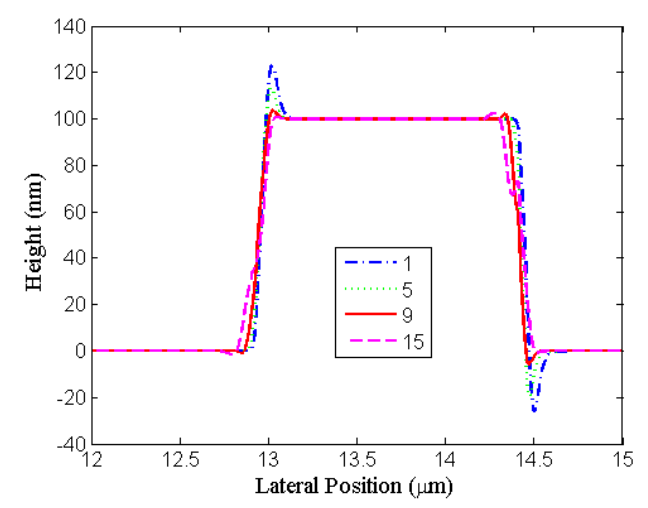

b)

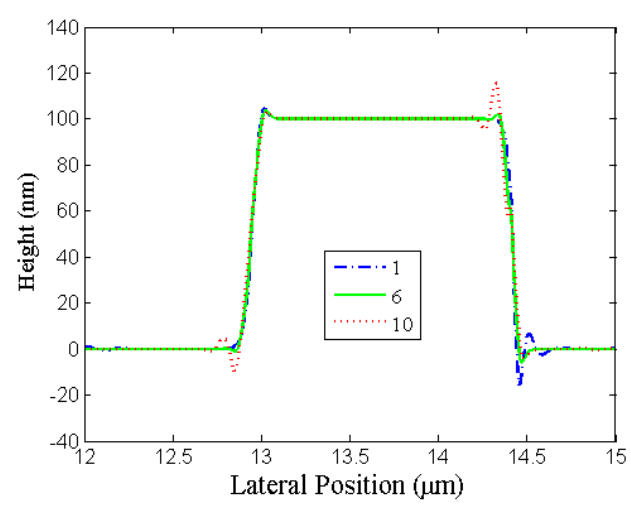

Figure 10. The effect of a) nq when $n b=6$ and b) $n b$ when $n q=9$ on the scan performance.

In Figure 11a, the scan performance of the $\mathrm{RC}(\mathrm{nq}=9$ and $\mathrm{nb}=6)$ is compared to that of the stand-alone PI controller at the scan speed of $3 \mu \mathrm{m} / \mathrm{s}$. Considering the structure given in Figure 5, we first tune the PI controller for the best performance and then turn on the RC for the comparison. As shown in the figure, the stand-alone PI controller responds to the changes in step profile with a delay whereas the RC can respond instantly. Though the delay is small at this speed, the relative improvement in scan error is $75 \%$ when the RC controller is used instead of the stand-alone PI controller. To compare the performance of the stand-alone PI with that of the RC at higher scan speeds, we also performed scans at the speed of $21 \mu \mathrm{m} / \mathrm{s}$ (see Figure 11b). Compared to the standalone PI controller, the RC reduces the scan error by $58 \%$. The scan errors for the scan speeds of 3 $\mu \mathrm{m} / \mathbf{s}$ and $21 \mu \mathrm{m} / \mathrm{s}$ are summarized in Table 1. In order to achieve even better tracking results, we show that the bandwidth of the Q filter in the RC can be adjusted. When the bandwidth of the RC controller is increased (see the discussion in Section 3.4), we observed that the relative difference in scan error is increased to $64 \%$ (see the scan profiles for $\mathrm{Q}_{1}$ and $\mathrm{Q}_{2}$ in Figure $11 \mathrm{~b}$ ). 
a)
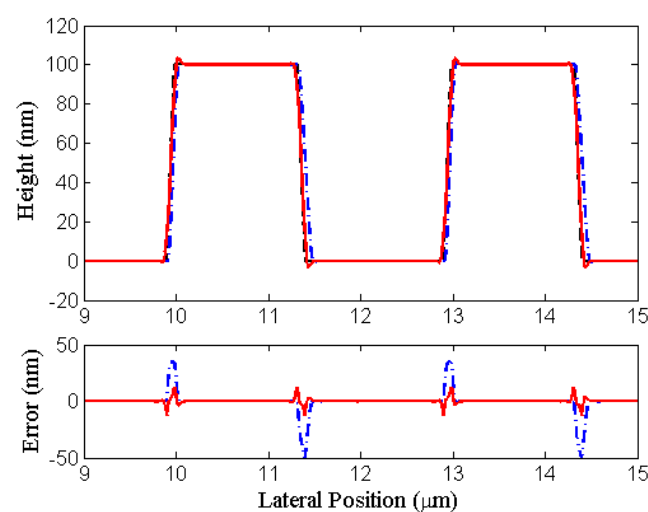

b)
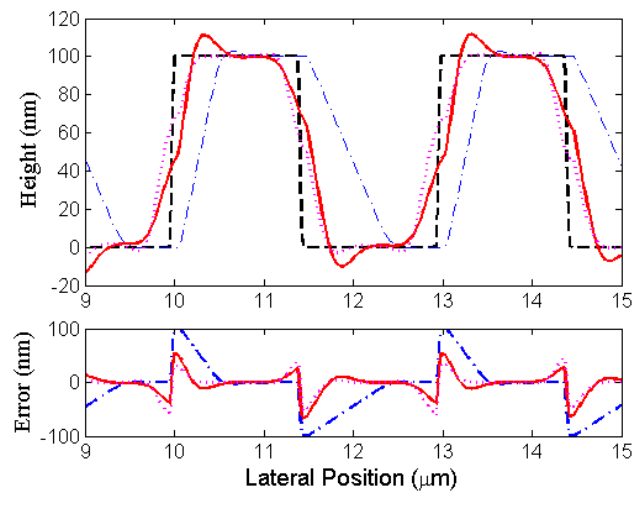

Figure 11. The scan results for the scan speed of a) $3 \mu \mathrm{m} / \mathrm{s}$ and b) $21 \mu \mathrm{m} / \mathrm{s}$ : the surface being scanned (dashed black), the results obtained by the PI controller (dash-dotted blue), the $\mathrm{RC}\left(\mathrm{Q}_{1}\right.$, solid red) and the $\mathrm{RC}$ with an extended bandwidth $\left(\mathrm{Q}_{2}\right.$, dotted red).

One of the most important benefits of the simulation environment is that the tapping forces can be calculated from the force model directly and used as a performance measure. Since our simulations are in tapping mode, both the maximum and the deviation of instantaneous tapping forces from the nominal value are considered for the evaluation of the scan results. The maximum tapping forces must be reduced to prevent damage to the probe and the sample, especially when biological samples are scanned. The deviation of instantaneous tapping forces from the nominal value is an indicator of the scan quality. The instantaneous tapping forces as a function of lateral position are shown in Figure 12a and Figure $12 \mathrm{~b}$ for the scan speeds of $3 \mu \mathrm{m} / \mathrm{s}$ and $21 \mu \mathrm{m} / \mathrm{s}$ respectively. The quantitative analysis shows that the maximum tapping force at the contact (see the instants of contact and no-contact in Figure 12) for the scan speed of $3 \mu \mathrm{m} / \mathrm{s}$ and $21 \mu \mathrm{m} / \mathrm{s}$ is reduced by $1 \%$ and $87 \%$ (and 95\% with the extended bandwidth), respectively, when the RC is used instead of the stand-alone PI controller. Moreover, the total deviation from the nominal force value is decreased by $95 \%$ and $93 \%$ (and 98\% with extended bandwidth) for the scan speeds of $3 \mu \mathrm{m} / \mathrm{s}$ and $21 \mu \mathrm{m} / \mathrm{s}$, respectively. The maximum tapping forces and the deviation of the tapping forces from the nominal value for the scan speeds of $3 \mu \mathrm{m} / \mathrm{s}$ and $21 \mu \mathrm{m} / \mathrm{s}$ are summarized in Table 1 . 
a)

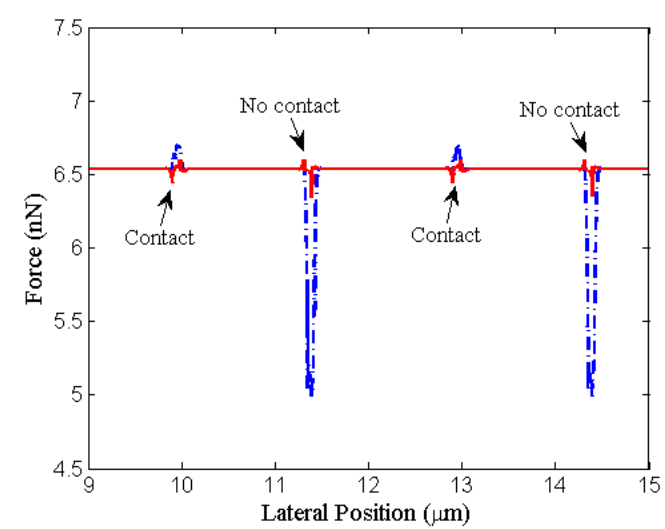

b)

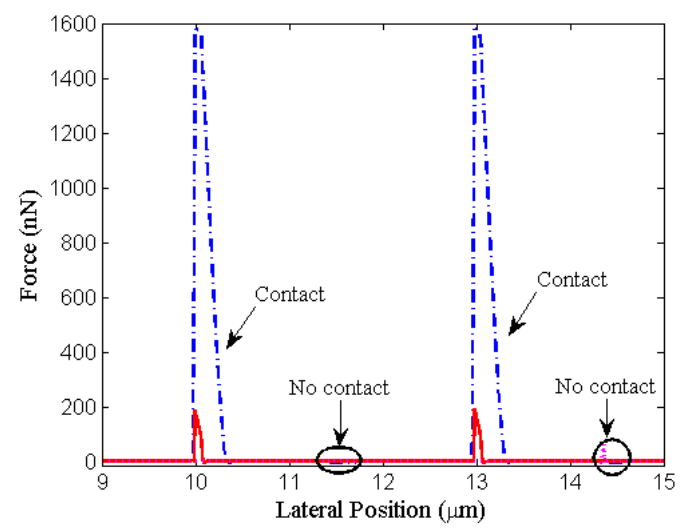

Figure 12. The tip-sample interaction forces as a function of lateral position for the scan speeds of a) 3 $\mu \mathrm{m} / \mathrm{s}$ and b) $21 \mu \mathrm{m} / \mathrm{s}$ under PI controller (dashed blue), RC (solid red), and RC with extended bandwidth (dotted red).

Table 1. The normalized scan error, the maximum tapping force and the deviation of the tapping forces from the nominal value for the scan speeds of $3 \mu \mathrm{m} / \mathrm{s}$ and $21 \mu \mathrm{m} / \mathrm{s}$.

\begin{tabular}{|c|c|c|c|c|}
\hline \multirow{2}{*}{} & \multicolumn{2}{|c|}{$3 \mathrm{um} / \mathrm{s}$} & \multicolumn{2}{c|}{$21 \mathrm{um} / \mathrm{s}$} \\
\cline { 2 - 5 } & PI & RC & PI & RC \\
\hline Scan Error (see Eq. 5) & $\mathbf{0 , 0 2 5 3}$ & $\mathbf{0 , 0 0 6 2}$ & $\mathbf{0 , 0 4 1 5}$ & $\mathbf{0 , 0 1 7 1}$ \\
\hline Max. Force (nN) & 6,7 & 6,6 & 1580 & 195 \\
\hline Deviation from Nominal Force (nN) & $\mathbf{0 , 2 3 3}$ & $\mathbf{0 , 0 1 0 3}$ & 322 & 21,3 \\
\hline
\end{tabular}

Furthermore, we compare the tracking performance of the $\mathrm{RC}$ to that of the stand-alone PI controller not only for a square wave, but also for two other wave forms: a triangular wave of amplitude $100 \mathrm{~nm}$ (Figure 13a) and a wave form made of two sinusoids having the wave lengths of $100 \mathrm{~nm}$ and $40 \mathrm{~nm}$ (Figure 13c). The results show that the RC outperforms the stand-alone PI controller in all cases (see the error profiles in Figures 13b and 13d). 
a)

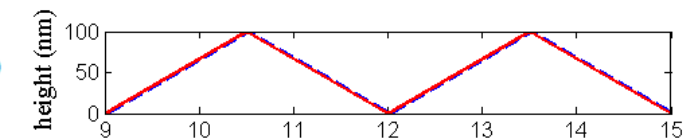

b)

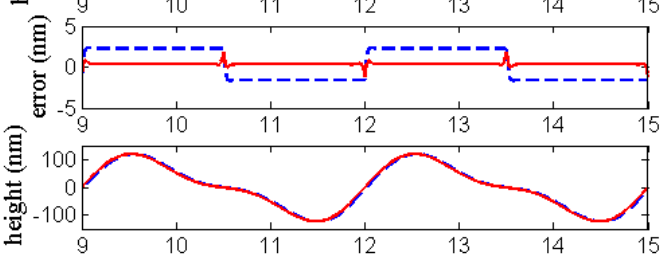

d)

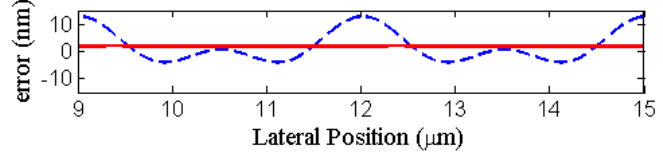

Figure 13. The tracking performance of the PI (blue dashed) and RC (red solid) for a) a triangular input and c) for an input composed of two sinusoids. The corresponding error profiles are shown in b) and d) respectively.

Finally, we further investigate the tracking performance and the stability of $\mathrm{RC}$ under various uncertainties by running simulation scenarios. The aim in this part is to question the scan performance of the RC when something unexpected happens. Since the AFM scan process is affected by many factors most of which are not easy to detect and eliminate, we believe that the performance of any new controller should be further investigated for possible structured and unstructured uncertainties existing in the system.

Scenario 1: The proposed RC assumes that the consecutive scan lines are similar to each other and takes a control action in the current scan line based on the error estimated for the previous scan line. However, if there is a significant difference between the consecutive scan profiles, the tracking performance of a typical RC will be poor since it can only react to this difference after one scan line is passed. In our implementation, an RC is connected in series with a standard PI controller such that the difference between the consecutive scan profiles can be compensated by the PI controller. In order to demonstrate the benefits of our approach, we design a scenario in which the step height is suddenly reduced from $100 \mathrm{~nm}$ to $10 \mathrm{~nm}$ (Figure 14). In this scenario, each step can be considered as one scan line for the sake of discussion. Initially, the stage tracks the $100 \mathrm{~nm}$ steps successfully, but when a sudden change in the step profile occurs, the repetition in the profile is broken. Then, the PI controller becomes more active and tracks the input signal until the repetition in the profile is established again. 
Note that the sudden reduction in the step height causes a peak in the scan profile due to the control signal carried on by the $\mathrm{RC}$ from the previous step is larger than the small value desired for the current step. The size of this peak can be reduced by adding a derivative term to the PI controller. We also note that such undesired peaks do not appear at all when there is less than $50 \%$ reduction in the amplitude. On the contrary, a sudden increase in the step size does not constitute such a problem since the control signal carried on by the RC from the previous line is small, and it is dissolved in the larger control signal generated by the PI controller.

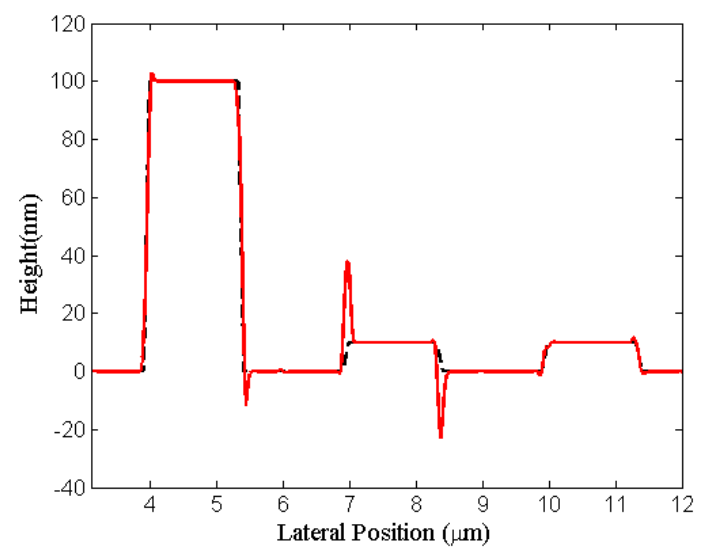

Figure 14. Our RC is designed as an add-on to a standard PI controller so that the PI controller can help with the tracking when the consecutive scan profiles are significantly different from each other. In the graph above, each step is considered as one scan line.

Scenario 2: An AFM probe can be damaged easily during tapping-mode AFM scanning, since it is in continuous contact with the sample surface. This results in a change in the mass and consequently the resonance frequency of the probe. If undetected, the free air amplitude of the defected probe decreases, which also causes a decrease in the magnitude of the error signal transmitted to the controller. Under these circumstances, the PI controller needs to be re-tuned whereas the RC can compensate for the drop in the magnitude of the error signal and does not require re-tuning. The following simulation scenario is developed to demonstrate this concept: Initially, the probe's amplitude is set to the $70 \%$ of the free air amplitude $\left(A_{\text {free }}=67 \mathrm{~nm}\right)$. Then, the free air amplitude is suddenly reduced to $A_{\text {free }}=57 \mathrm{~nm}$ without changing the set amplitude. The resulting scan profile is shown in Figure 15. It is evident that 
the PI controller suffers from the error saturation at the falling edges of the steps whereas the RC successfully tracks the surface profile.

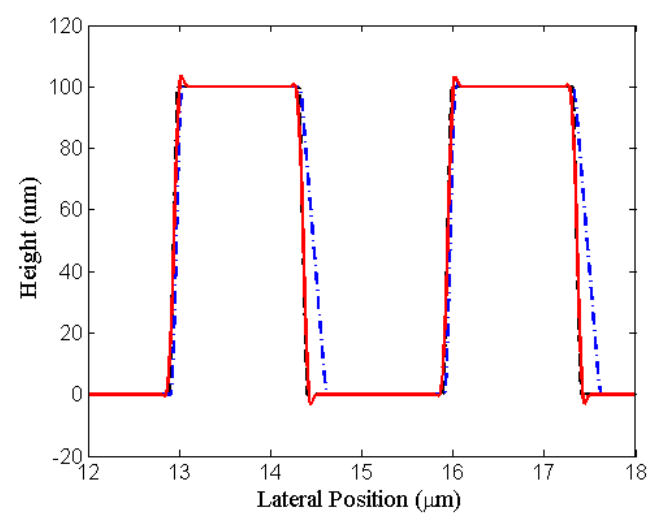

Figure 15. The scan profile for a damaged probe: the actual surface (dashed black), the PI controller (dash dotted blue) and the RC (solid red).

Scenario 3: The interactions of a stiff and a soft probe with the sample surface are investigated in section 4. As concluded, a soft probe can stick to the sample surface lot easier than a stiff one. When the soft probe sticks to the sample surface, a large error occurs and the controller generates a large control signal to move the stage downwards. This action causes undesired peaks in the scan profile. However, if the controller keeps the probe away from the sticking regime during the scan process (see Figure 8), which is determined by the tip-sample separation distance, the undesired peaks can be avoided. Since the RC takes a control action based on the scan results of the previous scan line, the sticking behavior is handled better. In Figure 16, we show that the soft probe sticks to the surface at the beginning of each step under the PI controller, causing an overshoot, which does not occur under the RC. 


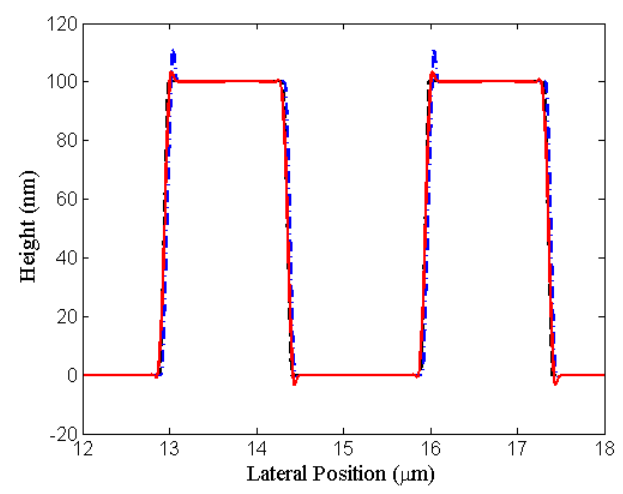

Figure 16. The scan performance of a soft probe under the PI controller (dash dotted blue) and the RC (solid red).

\section{Scanning Experiments}

Nano scanning experiments are performed with our home-made AFM setup to validate the results of the numerical simulations. A calibration grating (Micro-Masch TGZ02) consisting of steps with 3 $\mu \mathrm{m}$ pitch and $82.5 \mathrm{~nm}$ height $( \pm 1.5 \mathrm{~nm})$ is scanned. The AFM probe is excited at its resonance frequency $(353.8 \mathrm{kHz})$ with a free air vibration amplitude of $\mathrm{A}_{\text {free }}=52 \mathrm{~nm}$ (note that the set amplitude is equal to $70 \%$ of the free air amplitude). The scanning experiments are performed at the scan speeds of $3 \mu \mathrm{m} / \mathrm{s}$ and $21 \mu \mathrm{m} / \mathrm{s}$ (Figures 17 and 18 respectively). For the scans performed under the RC, nq and nb are set to 9 and 6 respectively based on the results of the simulations presented earlier (Figure 9). At a first glance, the improvement achieved by the RC is not obvious for the scan speed of $3 \mu \mathrm{m} / \mathrm{s}$ (compare Figures 17a and 17c), but the standard PI controller follows the desired profile with a delay as observed in the simulations. The tracking error due this delay is shown in Figure 17b (compare it with Figure 17d). Since the PI controller can track the surface with a delay, the probe sticks to the rising edge of each step, causing a sudden jump in the error signal. The same phase delay also causes a sudden drop in the error signal on the falling edge of each step. As the scan speed increases to 21 $\mu \mathrm{m} / \mathrm{s}$, the phase delay increases further and the difference between the profiles scanned by the standalone PI controller and the RC becomes more obvious (Figure 18). Moreover, on the rising edges of the steps, the PI controller suffers from the sticking behavior of the probe, which causes a large overshoot, while on the falling edges, the PI controller suffers from error saturation, which limits its 
response rate. However, both problems are reduced when the RC is used. The performance of the RC becomes even better if the bandwidth of the Q filter is doubled (the last row in Figure 18). The results of the scanning experiments performed with our AFM set-up show that the proposed RC significantly outperforms the conventional PI controller. Compared to the stand-alone PI controller, the scan error and the average tapping forces are reduced by $66 \%$ and $58 \%$, respectively when the scan speed is increased by 7 -fold [26].
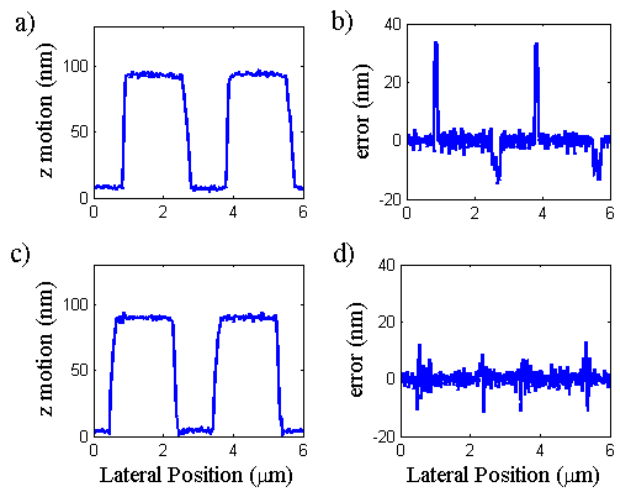

Figure 17. The scan profile and error signal for the scan speed of $3 \mu \mathrm{m} / \mathrm{s}$ under the standalone PI controller (a, b) and the RC (c, d).

a)

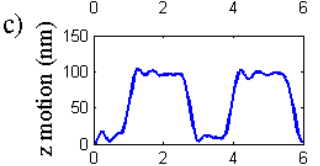

e)
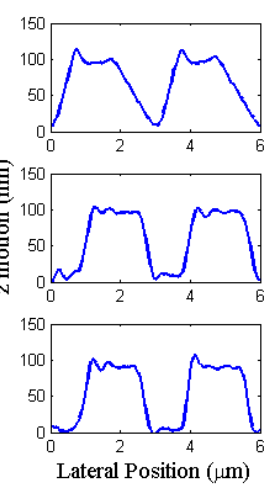

b)

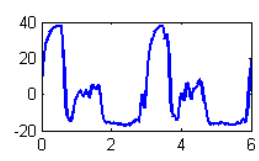

d)

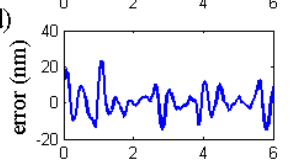

f)

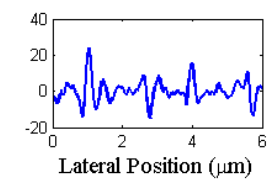

g)

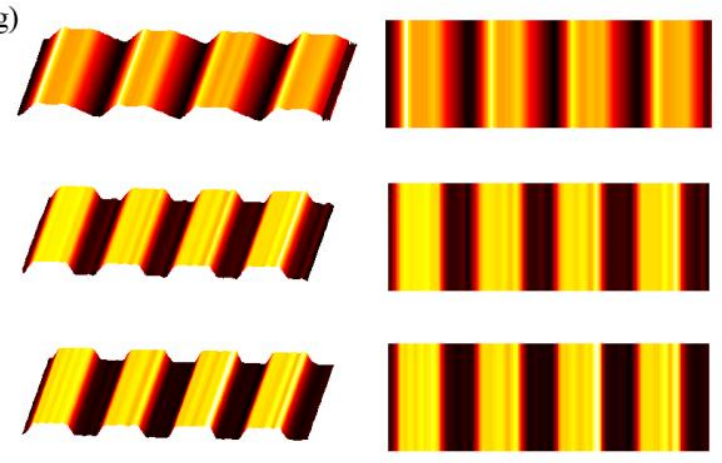

Figure 18. The scan profile and error signal for the scan speed of $21 \mu \mathrm{m} / \mathrm{s}$ under the stand-alone PI controller ( $a, b)$, the RC (c, d), and the RC with extended bandwidth (e, f). The 3D representation of the same scan profiles are shown in $(\mathrm{g})$. 


\section{Discussion and Conclusion}

We investigated the use of RC in AFM scanning through scan experiments and numerical simulations. We showed that RC improves the image quality and reduces the tapping forces when compared to a stand-alone PI controller. Although the improvement in the image quality cannot be observed directly from the scan profiles at low scanning speeds, it is much clearer when the scan speed is increased. The RC is superior to the stand-alone PI controller because it is designed to compensate for the phase delays better when the input signal is repetitive. The positive effects of this phase cancellation are more obvious at high scan speeds since the problems causing a delay in the response of the stage, sticking of the probe to the surface and the error saturation, occur more frequently. The cancellation of phase delays is possible with the help of memory loop in the RC, which utilizes the profile of the previous scan line to take a control action in the current scan line. It is also observed in the simulations that the proposed implementation of the $\mathrm{RC}$ as an add-on to a PI controller is effective when significant differences exist between the profiles of consecutive scan lines. However, such a large difference is unexpected in real AFM scans if sufficiently small advances are made along the yaxis.

The results of our numerical simulations also support the AFM experiments performed in our setup. Although numerical simulations have been performed in the past to investigate the cantilever dynamics and tip-sample interactions, only a few recent studies have focused on the simulation of the whole scan process $[27,28]$. Since it is almost impossible to repeat a scan experiment under exactly the same conditions in real world settings due to variations in humidity, electrical noise, ground vibrations, temperature, etc, developing numerical simulations to test the performance of a new controller is important. Moreover, even if the experimental conditions are fixed, the probe tip or the sample surface may get damaged in time, affecting the scan results adversely. All these factors make it difficult to compare the results of experimental scans quantitatively under different scan settings. On the other hand, we, for example, easily repeated the scan experiments in Simulink/Matlab environment to construct iso-curves of constant error for different values of sample advance parameters (nq and nb) of the RC to find their optimum values. In fact, those values $(\mathrm{nq}=9$ and $\mathrm{nb}=6)$ also worked well for 
the scanning experiments performed in our AFM set-up. In addition, we readily accessed all the outputs of the simulations, some of which are not directly measurable in physical experiments (e.g. the magnitude of the tapping forces shown in Figure 12). Finally, we also designed simulation scenarios to investigate the robustness of the RC under various uncertainties. We showed that the RC does not require re-tuning of the parameters under the simulated uncertainties.

\section{References}

[1] Ando T, Kodera N, Takai E, Maruyama D, Saito K and Toda A 2001 A high-speed atomic force microscope for studying biological macromolecules Proceedings of the National Academy of Sciences, 9812468.

[2] Rost M J, Crama L, Schakel P, Tol E, Velzen-Williams G B E M, Overgauw C F, Horst H, Dekker H, Okhuijsen B, Seynen M, Vijftigschild A, Han P, Katan A J, Schoots K, Schumm R, Loo W, Oosterkamp T H, and Frenken, J W M 2005 Scanning probe microscopes go video rate and beyond Rev. Sci. Instrum.76 053710

[3] Schitter G, Aström G, DeMartini B E, Thurner P J, Turner K L and Hansma P K 2007 Design and Modeling of a High-Speed AFM-Scanner IEEE Transaction on Control System Technology 15(5) 906

[4] Fukuma T Okazaki Y Kodera N Uchihashi T and Ando T 2008 High resonance frequency force microscope scanner using inertia balance support Appl. Phys. Lett. 92243119

[5] Clayton G M., Tien S., Leang K K., Leang, Zou, Q, and Devasia, S. 2009 A Review of Feedforward Control Approaches in Nanopositioning for High Speed SPM ASME Journal of Dynamic Syst ems, Measurement and Control, 131 (6)

[6] Devasia S., Eleftheriou, E., Moheimani, S.O.R. 2007 A Survey of Control Issues in Nanopositioning IEEE Transactions on Control Systems Technology, 15 (5), 802-823.

[7] Salapaka S. and Salapaka M. V., 2008 Scanning Probe Microscopy - A Controls Systems Perspective on Nanointerrogation IEEE Control Systems Magazine, 65-83, April.

[8] Croft D, Shed G and Devasia S 2001 Creep, Hysteresis, and Vibration Compensation for Piezoactuators: Atomic Force Microscopy Application ASME Journal of Dynamic Systems, Measurement, and Control $\mathbf{1 2 3} 35$

[9] Zou Q, Leang K K, Sadoun E, Reed M J, Devasia S 2004 Control Issues In High-Speed AFM for Biological Applications: Collagen Imaging Example Asian Journal of Control 6164

[10] Leang K K and Devasia S 2007 Feedback-Linearized Inverse Feedforward for Creep, Hysteresis, and Vibration Compensation in AFM Piezoactuators IEEE Transactions on Control Systems Technology 15927

[11] Hung S K, Hwu E T, Hwang I S, Fu L C 2006 Postfitting Control Scheme for Periodic Piezoscanner Driving Japanese Journal of Applied Physics 451917 
[12] Wu Y and Zou Q 2007 Iterative Control Approach to Compensate for Both the Hysteresis and the Dynamics Effects of Piezo Actuators IEEE Transactions on Control Systems Technology 15936

[13] Kim K S, Zou Q and Su C 2007 Iteration-based Scan-Trajectory Design and Control with Output Oscillation Minimization: Atomic Force Microscope Example Proceedings of the American Control Conference 4227

[14] Li Y and Bechhoefer J 2008 Feedforward Control of a Piezoelectric Flexure Stage for AFM Proceedings of the American Control Conference 2703

[15] Salapaka S, Sebastian A, Cleveland J P and Salapaka M V 2002 High bandwidth nanopositioner: A robust control approach Rev. Sci. Instrum. 73093232

[16] Leang K K, Devasia S. 2005 Design of hysteresis-compensating iterative learning control for piezo-positioners: application to atomic force microscopes. Mechatronics 16(3):141-58.

[17] Helfrich B E, Lee C, Bristow, D A, Xiao, X H, Dong J, Alleyne A G, Salapaka, S M, Ferreira P M 2010 Combined $\mathrm{H}_{\infty}$-Feedback Control and Iterative Learning Control Design With Application to Nanopositioning Systems IEEE Transactions on Control Systems Technology, 18(2) 336.

[18] Bhikkaji B and Moheimani S O R 2009 Fast scanning using piezoelectric tube nanopositioners: A negative imaginary approach IEEE/ASME International Conference on Advanced Intelligent Mechatronics 274

[19] Aridogan U, Shan Y and Leang K K 2009 Design and Analysis of Discrete-Time Repetitive Control for Scanning Probe Microscopes ASME Journal of Dynamic Systems, Measurement, and Control 131061103

[20] Lin, C., Chen, P. 2011 Precision tracking control of a biaxial piezo stage using repetitive control and double-feedforward compensation Mechatronics, 21 (1), 239-249.

[21] Merry R J E, Ronde M J C, Molengraft R, Koops K R and Steinbuch M 2010 Directional Repetitive Control of a Metrological AFM IEEE Transaction on Control System Technology, doi: $\underline{10.1109 / T C S T .2010 .2091642}$

[22] Schitter G, Allgöwer F and Stemmer A 2003 A new control strategy for high-speed atomic force microscopy Nanotechnology 15108

[23] Kodera N and Sakashita M 2006 Dynamic proportional-integral-differential controller for highspeed atomic force microscopy Rev. Sci. Instrum. 77083704

[24] Fujimoto H and Oshima T 2008 Nanoscale Servo Control of Contact-mode AFM with Surface Topography Learning Observer IEEE International Workshop on Advanced Motion Control 568

[25] Wu Y, Zou Q and Su C 2009 A Current Cycle Feedback Iterative Learning Control Approach for AFM Imaging IEEE Transactions on Nanotechnology 8515

[26] Necipoglu S, Cebeci S, Has Y, Guvenc L, Basdogan C, 2011 A Robust Repetitive Controller for Fast AFM Imaging, to appear in IEEE Transactions on Nanotechnology, doi: 10.1109/TNANO.2011.2106797

[27] Varol A, Gunev I, Orun B and Basdogan C 2008 Numerical simulation of nano scanning in intermittent-contact mode AFM under Q control Nanotechnology 19075503 
[28] Kowaleski T and Legleiter J 2006 Imaging stability and average tip-sample force in tapping mode atomic force microscopy Journal of Applied Physics 99064903

[29] Guvenc B A and Guvenc L 2006 Robust repetitive controller design in parameter space ASME Journal of Dynamic Systems, Measurement, and Control, 128406

[30] Srinivasan K and Shaw F R 1991 Analysis and Design of Repetitive Control Systems Using the Regeneration Spectrum ASME Journal of Dynamic Systems, Measurement, and Control 113216

[31] Gunev I, Varol A, Karaman S and Basdogan C 2007 Adaptive Q control for tapping-mode nanoscanning using a piezoactuated bimorph probe Rev. Sci. Instrum. 78043707 